\title{
Translation, Adaptation and Validation of a Chinese Medicine Questionnaire to Brazilian Portuguese
}

\author{
Ciro Blujus dos Santos Rohde ${ }^{1,2^{*}}{ }^{(0)}$, Lukas Blumrich ${ }^{3}(\mathbb{0})$, Pedro Oliveira Dias Martins ${ }^{3}$ (), \\ Fernanda Babler 4 (), Wu Tu Hsing 5 (), Hermano Tavares 2 다
}

${ }^{1}$ Service of Phytotherapy, Medicinal Plants and Acupuncture of the Impulse Control Disorders and Behavioral Addictions Outpatient Unit, Institute and Department of Psychiatry, University of São Paulo, São Paulo, Brazil

${ }^{2}$ Impulse Control Disorders and Behavioral Addictions Outpatient Unit, Institute and Department of Psychiatry, University of São Paulo, São Paulo, Brazil

${ }^{3}$ School of Medicine, University of São Paulo, São Paulo, Brazil

${ }^{4}$ Institute of Radiology, Hospital of Clinics, University of São Paulo, São Paulo, Brazil

${ }^{5}$ Center of Acupuncture of the Institute of Orthopedics and Traumatology of the Hospital of Clinics, School of Medicine,

University of São Paulo, São Paulo, Brazil

Email: *cirobsr@gmail.com, ${ }^{\star}$ ciro.rohde@hc.fm.usp.br, lukas.blumrich@yahoo.com.br, pedro.martins@fm.usp.br,

fbabler@gmail.com,wu@fm.usp.br, hermanot@uol.com.br

How to cite this paper: dos Santos Rohde, C.B., Blumrich, L., Martins, P.O.D., Babler, F., Hsing, W.T. and Tavares, H. (2021) Translation, Adaptation and Validation of a Chinese Medicine Questionnaire to Brazilian Portuguese. Open Journal of Applied Sciences, 11, 1149-1156.

https://doi.org/10.4236/ojapps.2021.1110086

Received: September 21, 2021

Accepted: October 26, 2021

Published: October 29, 2021

Copyright $\odot 2021$ by author(s) and Scientific Research Publishing Inc. This work is licensed under the Creative Commons Attribution International License (CC BY 4.0).

http://creativecommons.org/licenses/by/4.0/

\begin{abstract}
The Constitution in Chinese Medicine Questionnaire [CCMQ] organizes 9 body constitutions according to Traditional Chinese Medicine [TCM]. The translation was realized by a Medical Doctor [MD] specialized in TCM and the back translation by an english-speaking MD. Comprehensibility assessment was performed ( $\mathrm{N}=40$, main scores ranged from 4.20 to 4.95). On the test-retest reliability Cronbach's alpha was 0.920 in the first application and 0.943 in the second, the intraclass correlation scores ranged between good and excellent $(0.6->0.80)$. The results show that the adaptation of the CCMQ is valid and ready to be used.
\end{abstract}

\section{Keywords}

Traditional Chinese Medicine, Body Constitution, Cross-Cultural, Questionnaire, Portuguese

\section{Introduction}

The Chinese concept of body constitution is a model that comprehends many aspects of one individual's health [1]. Constitution (ti-zhi) is a widely used term 
in Traditional Chinese Medicine [TCM], and implies the description of the quality of the body. The theory of body constitutions was developed to better understand human health and physical and mental aspects related to disease development and course [2] [3]. To improve national health management, the Chinese government created a project to stablish a normative system of body constitutions according to TCM based in classificatory questionnaires [4]. The reproducibility, reliability and validation of the questionnaire in development were evaluated and published by the Chinese Association of Chinese Medicine in 2009 as the "Constitution in Chinese Medicine Questionnaire" [CCMQ] [5]. Its reliability and validation were evaluated with more than 20 thousand people in China in 9 distinct geographic districts [6] [7].

Since its development, CCMQ was translated and validated to different languages and communities [8] [9] [10]. The practical application of the CCMQ range from academic learning to preventive healthcare projects and both clinical research and approach to patients [11] [12]. Recent research points to correlations between the TCM body constitution and some diseases as metabolic syndrome, depression and other chronic diseases [13] [14] [15].

Recent data show that TCM plays an important role on treatment and disease prevention [16] [17] [18]. In Brazil, acupuncture and TCM are recognized as valid medical approaches by the Ministry of Health [19]. The goal of the present study was to report on preliminary data about the cultural adaptation and validation of the CCMQ for Brazilian Portuguese. The translation, adaptation, and validation of CCMQ to Brazilian Portuguese can improve acupuncture and TCM teaching and clinical practice in Brazil and other Portuguese-speaking countries, and enables new research projects on this subject.

\section{Methods}

For the validation of the CCMQ for Brazilian Portuguese, we adopted a sixstep procedure, following the proposals of Conti et al. [20], Teixeira et al. [21] e Toledo et al. [22] for validation of English questionnaires to Brazilian Portuguese.

\subsection{Step One: Translation}

The first translation of the English version into Brazilian Portuguese was provided by the first author (CBSR) based on a previously validated English version from Jing and colleagues [23].

\subsection{Step Two: Back-Translation}

The translated version was back-translated into English by a native Englishspeaking doctor (FB), familiar with the TCM concepts.

\subsection{Step Three: Peer Review and Evaluation of Semantic Equivalency by Experts}

The first and the senior author of the current report (CBSR and HT, respective- 
ly) reviewed the semantic equivalence between versions, having correct conceptual equivalence between versions as the main goal.

\subsection{Step Four: Instrument Evaluation through a Sample of Students, by Evaluating Their Understanding Level}

The second version of the translated questionnaire was then presented to 40 medical students from the School of Medicine of the University of São Paulo (Faculdade de Medicina da Universidade de São Paulo) for the comprehensibility evaluation via an online platform. The students were aware of the study's goal, and could only access the questionnaire after accepting an informed consent form. Also, the principal of the School of Medicine of the University of São Paulo signed a consent form for the students to be accessed by the researchers. An adapted verbal-numeric questionnaire was used [20] [21] [22], by which the subjects could rate the comprehensibility of the item in a Likert-like scale ranging from 0 ("I didn't understand anything") to 5 ("I understood perfectly and do not have questions"). After each item, a space was also made available for suggestions and comments. For the comprehensibility assessment, means of the comprehension scores for each item were calculated, and the Wilcoxon signedrank test was performed. The items with a mean score of less than 4 and the items with the lowest position in the rank test (if a significant difference were to be found) would be reviewed and eventually submitted to a new evaluation by the authors. A cutoff of $80 \%$ of "good" or "excellent" scores was also established to determine which items should be reviewed.

\subsection{Step Five: Analysis of the Instrument's Internal Consistency}

The internal consistency of the translated version was assessed by calculating Cronbach's alpha applied to the responses obtained with the second application of the questionnaire. The test-retest reliability assessment was performed with another 40 medical students from the same institution, contacted to answer the questionnaire twice via the same online platform, with an interval of two weeks.

\subsection{Step Six: Test-Retest Reliability}

For this final step, the questionnaire was answered twice by the same group of students, with an interval of two weeks between each assessment. The assessment of test-retest reliability was made using the intraclass correlation analysis method (ICC), which is best indicated for continuous variables [24], by comparing the nine scores for each body constitution according to the scoring-key of the original instrument obtained with the first completion of the CCMQ with the scores of the second application. Analyses were performed with SPSS 22.0 software (SPSS Inc., Chicago, IL, USA).

\section{Results}

\subsection{Transcultural Translation and Adaptation}

A total of 6 items presented discrepancies between the original and the back- 
translated version, but only two of those were considered important by the authors (CBSR and HT) designated to compare and evaluate the back-translated version in relation to the original version. For one of these items, the authors suggested a specific adaptation regarding the expression "hot flashes", because a direct translation into the corresponding wording did not describe the symptom in its usual wording for Brazilian Portuguese. The translation for the other item analyzed added the expression "breathe deeply" to clarify the meaning of "sigh", but this change was considered misleading, as this expression can be related not only to emotional questions (as originally intended) but also to respiratory problems. The suggestions made by these authors were discussed with and approved by the others.

\subsection{Comprehensibility Assessment}

The comprehensibility mean scores of the CCMQ items ranged from 4.20 to 4.95. The Wilcoxon signed-rank test identified three levels or blocks of comprehensibility. The first block comprised 43 items, headed by item \#1.6, with a mean score of 4.88. Then, there was a second block of 20 items, headed by item \#6.5, they all differed from the leading comprehensibility score, with a mean score of 4.67. Finally, there was a third block comprising items \#4.1, \#4.2, \#5.2 and \#5.7 with the lowest comprehensibility scores, all differing from level two highest score, with a mean score of 4.33 . Table 1 shows the main outcomes.

Only two items scored below the previously established cutoff for comprehension scores. Both (Items 4.1 and 5.2) had an average of $77.5 \%$ of good or excellent rating. In fact, both items were just one score 4 or 5 short to achieve the required cutoff. These items were reviewed and discussed by the researchers, but it was concluded that there was no safe way to make the text clearer, at the risk of important conceptual changes.

Table 1. Comprehensibility scores and evaluation.

\begin{tabular}{|c|c|c|c|c|c|}
\hline \multirow{2}{*}{ Comprehensibility } & \multirow{2}{*}{ Item ranking } & \multicolumn{2}{|c|}{ Comprehensibility } & \multicolumn{2}{|c|}{ Wilcoxon's test } \\
\hline & & Excellent (\%) & Good (\%) & $\mathrm{Z}$ & $\mathrm{p}$ \\
\hline Best ranked item within level $1^{\mathrm{a}}$ & Item $\# 1.6^{\mathrm{d}}$ & 95 & 5 & - & - \\
\hline Worst ranked item within level 1 & Item $\# 7.2$ & 85 & 7.5 & -1.890 & 0.059 \\
\hline Best ranked item within level $2^{\mathrm{b}}$ & Item $\# 6.5^{\mathrm{e}}$ & 82.5 & 10 & -2.530 & $\underline{0.011}$ \\
\hline Worst ranked item within level 2 & Item \#4.7 & 75 & 17.5 & -0.884 & 0.376 \\
\hline Best ranked item within level $3^{c}$ & Item $\# 5.7^{\mathrm{f}}$ & 62.5 & 22.5 & -2.360 & $\underline{0.018}$ \\
\hline Worst ranked item within level 3 & Item \#4.1 & 57.5 & 20 & -1.049 & 0.294 \\
\hline
\end{tabular}

${ }^{a}$ Level 1 items in ranking order: \#1.6, \#2.2, \#3.6, \#3.5, \#8.7, \#7.4, \#1.7, \#9.1, \#2.6, \#2.3, \#7.5, \#1.1, \#3.7, \#1.5, $\# 1.3$, \#9.8, \#9.3, \#9.2, \#8.2, \#7.7, \#5.3, \#4.5, \#8.4, \#7.6, \#6.2, \#5.6, \#5.4, \#3.8, \#9.7, \#4.4, \#6.7, \#4.3, \#3.4, \#3.2, $\# 5.1, \# 8.3, \# 2.7, \# 3.3, \# 1.2, \# 8.1, \# 6.1, \# 2.8, \# 7.2$; bevel 2 items in ranking order: \#6.5, \#2.5, \#9.6, \#6.3, \#2.4, \#8.5, \#2.1, \#9.5, \#4.6, \#3.1, \#9.4, \#6.6, \#7.1, \#1.4, \#5.5, \#6.4, \#4.8, \#7.3, \#8.6, \#4.7; ' Level 3 items in ranking order: \#5.7, \#5.2, \#4.2, \#4.1; ${ }^{\mathrm{d}}$ Reference score for comparison with remaining items from level 1 and first

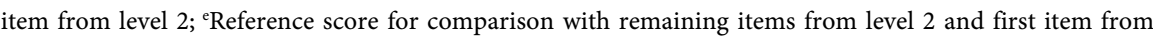
level 3; ${ }^{\mathrm{f}}$ Reference score for comparison with the remaining items from level 3. 


\subsection{Test-Retest Reliability}

The mean completion time for the first application was 12 minutes and 48 seconds.

Cronbach's alpha for the entire instrument was 0.920 in the first application and 0.943 in the second application. The alpha coefficients for each subscale from the CCMQ were considerably lower ranging from 0.53 to 0.88 . The majority were slightly above 0.60 (moderate); three fell below 0.60 (low): Stagnant blood, Yin-deficiency and Neutral; and only Stagnant-qui alpha was above 0.80 (very good). The intraclass correlation scores for all CCMQ subscores ranged between good and excellent $(0.6->0.80)$. Table 2 presents a summary of the main outcomes.

\section{Discussion}

The results show that the adaptation of the CCMQ to Brazilian Portuguese performed in this study is valid and ready to be used. The analyzes of the comprehensibility scores allowed a very careful assessment of the potentially confusing items. There was no item with a mean comprehensibility score below 4 . The two items with less than $80 \%$ of "good" or "excellent" ratings would be above this cutoff with the change of a single evaluation, thus a decision was made to keep the actual item versions. However, in a posterior reflection, we could not discard the possibility that maybe the translation of these items deserved a review. Our best guesses would be the word "stuffiness" from item \#4.1 was misinterpreted and deserved another translation to better indicate constipation of the chest and stomach as originally intended; whereas with item \#5.2 a more subtle problem may have happened regarding the concept of "having acne or sore easily", perhaps a more connotative translation could better imply the original concept of experiencing acnes and sores as a passive process rather than something actively sought. In both cases, these adaptations can be provided with slight language adjustments in Portuguese, and they must be tested in the ensuing works of the CCMQ in Brazil.

The value of Cronbach's alpha for the entire questionnaire was very high in both applications, what suggests great reliability of the instrument. In the case of the coefficients for the subscales, however, the values were considerably lower, with only one of these being classified as very good, which suggests that our adapted version may have a structure more complex than a one-factor model, as it happens with its original version. Nonetheless, the study was not designed for the assessment of its domains or underlying dimensions, which requires a factorial analysis and a bigger sample. In fact, testing the underlying structure of the symptoms listed in the CCMQ, according to the theoretical TCM framework, in a larger community sample from a diverse sociocultural milieu would be the natural following step, as it may yield precious insights into theoretical model of the TCM itself, i.e., how much of it can be automatically transposed to a different cultural milieu. 
Table 2. Test-retest reliability results.

\begin{tabular}{ccc}
\hline CCMQ Subscales & Cronbach's alpha $^{\mathrm{a}}$ & Test-retest intraclass Correlation Coefficient (ICC) $^{\mathrm{b}}$ \\
\hline Yang-deficiency & 0.692 & 0.619 \\
Yin-deficiency & 0.580 & 0.781 \\
Qi-deficiency & 0.623 & 0.888 \\
Phlegm-damp & 0.624 & 0.721 \\
Damp-heat & 0.630 & 0.827 \\
Stagnant-blood & 0.531 & 0.836 \\
Inherited-special & 0.758 & 0.861 \\
Stagnant-qi & 0.884 & 0.814 \\
Neutral & 0.532 & 0.798 \\
\hline
\end{tabular}

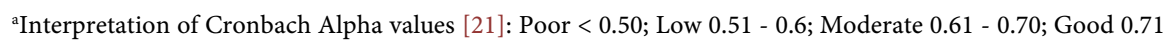
- 0.80; Very Good > 0.8; 'bnterpretation of ICC values [22]: Poor < 0.40; Fair 0.40 - 0.59; Good 0.60 - 0.74; Excellent $0.75-1.00$.

The analysis of the ICC coefficients shows that our version of the CCMQ has good to excellent test-retest reliability. Indeed, two out of nine subscores had ICCs within the "Good" range, and the other ICCs for the remaining scores were within the "Excellent" range.

\section{Limitations}

Both samples used in this study for comprehension and reliability analyzes were small and participation was on a voluntary basis, which may increase sample skewness. Besides, the subjects constituted a very specific subgroup of, i.e., Medicine School students, which could lead to further bias due to their peculiar profile and soon-to-be professional gaze upon symptom experience and appraisal.

\section{Conclusion}

The Brazilian Portuguese version of the CCMQ is the only one of its kind validated and ready to be used in future research endeavors, as well as to support the clinical practice of acupuncture and TCM in our country. Overall, the items translation was quite direct, but a continuous validation effort of instruments of the CCMQ, e.g., exploring its factorial structure, is necessary to further investigate the strengths and limitations of tradition-based medical models. So far, the evidence from the current report speaks in favor of the cross-cultural validity of the CCMQ.

\section{Source of Support}

Impulse Control Disorders and Behavioral Addictions Outpatient Unit, Institute and Department of Psychiatry, University of São Paulo, São Paulo, Brazil.

\section{Conflicts of Interest}

The authors declare no conflicts of interest regarding the publication of this paper. 


\section{References}

[1] Zhu, Y., Shi, H., Wang, Q., Wang, Y., Yu, X., Di, J., Zhang, X., Li, Y. and Yan, H. (2017) Association between Nine Types of TCM Constitution and Five Chronic Diseases: A Correspondence Analysis Based on a Sample of 2660 Participants. Evidence-Based Complementary and Alternative Medicine, 2017, Article ID: 9439682. https://doi.org/10.1155/2017/9439682

[2] Wang, J., Li, Y., Ni, C., Zhang, H. and Wang, Q. (2011) Cognition Research and Constitutional Classification in Chinese Medicine. The American Journal of Chinese Medicine, 39, 651-660. https://doi.org/10.1142/S0192415X11009093

[3] Jiang, Q.Y., Li, J., Zheng, L., Wang, G.H. and Wang, J. (2018) Constitution of Traditional Chinese Medicine and Related Factors in Women of Childbearing Age. Journal of Chinese Medical Association, 81, 358-365. https://doi.org/10.1016/j.jcma.2018.01.005

[4] Wang, Q., Zhu, Y.B., Xue, H.S. and Li, S. (2006) Primary Compiling of Constitution in Chinese Medicine Questionnaire. Chinese Journal of Clinical Rehabilitation, 10, 12-14.

[5] China Association of Chinese Medicine (2009) Classification and Identification of Constitution Theory of TCM (ZYYXH/T157-2009). World Journal of Traditional Chinese Medicine, 4, 303-304.

[6] Zhu, Y.B., Wang, Q. and Hideki, O. (2007) Evaluation on Reliability and Validity of the Constitution in Chinese Medicine Questionnaire (CCMQ). Chinese Journal of Behavioral Medical Science, 16, 651-654.

[7] Wang, Q. and Zhu, Y.B. (2009) Epidemiological Investigation of Constitutional Types of Chinese Medicine in General Population: Base on 21.948 Epidemiological Investigation Data of Nine Provinces in China. China Journal of Traditional Chinese Medicine and Pharmacy, 24, 7-12.

[8] Wong, W., Lam, C.L.K., Wong, V.T., Yang, Z.M., Ziea, E.T.C. and Kwan, K.L. (2013) Validation of the Constitution in Chinese Medicine Questionnaire: Does the Traditional Chinese Medicine Concept of Body Constitution Exist? Evidence-Based Complementary and Alternative Medicine, 2013, Article ID: 481491. https://doi.org/10.1155/2013/481491

[9] Jing, H., Wang, J., Wang, Q. and Bai, M. (2012) Applying Constitution in Chinese Medicine Questionnaire, Designed by Wang Qi (English Version) to Survey TCM Constitutions of the American and Canadian Caucasian in Beijing. China Journal of Traditional Chinese Medicine and Pharmacy, 27, 2417-2419.

[10] Zhu, Y., Origasa, H., Uebaba, K., Xu, F. and Wang, Q. (2008) Development and Validation of the Japanese Version of the Constitution in Chinese Medicine Questionnaire. Kampo Medicine, 59, 783-792. https://doi.org/10.3937/kampomed.59.783

[11] Mist, S., Ritenbaugh, C. and Aickin, M. (2009) Effects of Questionnaire-Based Diagnosis and Training on Inter-Rater Reliability among Practitioners of Traditional Chinese Medicine. The Journal of Alternative and Complementary Medicine, 15, 703-709. https://doi.org/10.1089/acm.2008.0488

[12] Cen, Y., Leung, R.K.K., Zhang, F., Jia, W., Zhang, J., Tan, X. and Xu, F. (2013) The Association between Yang-Deficient Constitution and Clinical Outcome of Highly Active Antiretroviral Therapy in People Living with HIV. Evidence-Based Complementary and Alternative Medicine, 2013, Article ID: 201857. https://doi.org/10.1155/2013/201857

[13] Feng, Y., Zheng, G. and Ling, C. (2012) The Investigation of the Correlation between Metabolic Syndrome and Chinese Medicine Constitution Types in Senior Re- 
tired Military Personnel of the People's Liberation Army. Chinese Journal of Integrative Medicine, 18, 485-489. https://doi.org/10.1007/s11655-011-0948-Z

[14] Liu, M., Jiang, Y., Wang, X., Liu, Q. and Wu, H. (2017) The Role of Rumination and Stressful Life Events in the Relationship between the Qi Stagnation Constitution and Depression in Women: A Moderated Mediation Model. Evidence-Based Complementary and Alternative Medicine, 2017, Article ID: 7605893. https://doi.org/10.1155/2017/7605893

[15] Liao, Y.C., Chou, C.Y., Chang, C.T., Li, T.S., Sun, M.F., Chang, H.H., Tsai, F.J. and Yen, H.R. (2017) Qi Deficiency i Associated with Depression in Chronic Hemodialysis Patients. Complementary Therapies in Medicine, 30, 102-106. https://doi.org/10.1016/j.ctim.2016.12.008

[16] Nielsen, A. and Wieland, L.S. (2019) Cochrane Reviews on Acupuncture Therapy for Pain: A Snapshot of the Current Evidence. Explore, 15, 434-439.

https://doi.org/10.1016/j.explore.2019.08.009

[17] Ni, X., Shergis, J.L., Zhang, A.L., Guo, X., Lu, C., Li, Y. and Xue, C.C. (2019) Traditional Use of Chinese Herbal Medicine for Insomnia and Priorities Setting of Future Clinical Research. The Journal of Alternative and Complementary Medicine, 25, 8-15. https://doi.org/10.1089/acm.2018.0249

[18] Li, Y., Li, X.H., Huang, X., Yin, L., Guo, C.X., Liu, C., He, Y.M., Liu, X. and Yuan, H. (2017) Individualized Prevention against Hypertension Based on Traditional Chinese Medicine Constitution. Theory Medicine, 96, e8513. https://doi.org/10.1097/MD.0000000000008513

[19] Brasil, Ministério da Saúde, Secretaria de Atenção à Saúde, Departamento de Atenção Básica (2006) Política nacional de práticas integrativas e complementares no SUS: Atitude de ampliação de acesso. Ministério da Saúde, Brasília.

[20] Sumintono, B. and Widhiarso, W. (2015) Aplikasi Pemodelan Rasch Pada Assessment Pendidikan. Trim Komunikata Publishing House, Cimahi.

[21] Cicchetti, D.V. and Sparrow, S.A. (1981) Developing Criteria for Establishing Interrater Reliability of Specific Items: Applications to Assessment of Adaptive Behavior. American Journal of Mental Deficiency, 86, 127-137.

[22] Toledo, E.L., et al. (2011) Adaptação transcultural da Massachusetts General Hospital (MGH) Hairpulling Scale para o idioma portugues (Brasil). Revista de Psiquiatria Clínica, 38, 178-183. https://doi.org/10.1590/S0101-60832011000500002

[23] Jing, H., Wang, J. and Wang, Q. (2015) Preliminary Compiling of English Version of Constitution in Chinese Medicine Questionnaire. Journal of Anhui University of Chinese Medicine, 34, 21-25.

[24] Shrout, P.E. and Fleiss, J.L. (1979) Intraclass Correlations: Uses in Assessing Rater Reliability. Psychological Bulletin, 86, 420-428. 